\title{
Er-doped tellurite waveguides for power amplifier applications
}

\author{
J. I.Mackenzie ${ }^{1 *}$, G.S.Murugan ${ }^{1}$, A.W.Yu ${ }^{2}$ and J.B.Abshire ${ }^{3}$ \\ ${ }^{1}$ Optoelectronics Research Centre, University of Southampton, Southampton SO17 1BJ, U.K. \\ ${ }^{2}$ Laser \& Electro-Optics Branch, NASA Goddard Space Flight Center, Greenbelt, MD 20771, U.S.A \\ ${ }^{3}$ Solar System Exploration Division, NASA Goddard Space Flight Center, Greenbelt, MD 20771, \\ U.S.A
}

Keywords: Optical Materials, Tellurite, Erbium, Erbium/Ytterbium, Laser amplifier, $\mathrm{CO}_{2}$

\begin{abstract}
:
Tellurite waveguides are promising candidates for high gain with broad-bandwidth, especially with Er-doping, which covers the telecommunications band and important spectral absorption features of atmospheric $\mathrm{CO}_{2}$. This study aimed at developing new Er,Yb-doped tellurite waveguide power amplifier modules suited to LIDAR measurements from space that will enable improved mapping of the concentration and distribution of $\mathrm{CO}_{2}$ in our atmosphere. A comparison of the optical properties of bulk and waveguide samples has been made, with double-clad waveguide devices, suited for high power diode pumping, currently under test.
\end{abstract}

\section{INTRODUCTION}

Tellurite waveguides are promising candidate materials for high optical-gain with broad spectral-bandwidth, which covers the telecommunications band and important spectral absorption features of atmospheric $\mathrm{CO}_{2}$, especially when doped with trivalent erbium ions. A relatively high index material (n 2) tellurite glasses are also strongly non-linear, with a nonlinear refractive index $n_{2}$ said to be up to two orders of magnitude higher than that of silica[1], opening up various possibilities in integrated photonic applications, such as, chip-based super-continuum or mode-locked lasers[2]. In this work, we present a review of our fabrication of new Er,Yb-doped multicomponent tellurite waveguide films aimed at developing power amplifier modules suited to LIDAR measurements from space, with a goal of improving the mapping of the concentration and distribution of $\mathrm{CO}_{2}$ in our atmosphere.

Comparing the optical properties of several compositions in bulk samples, we targeted ones that provided the best emission cross section characteristics around $1572 \mathrm{~nm}$ coincident with a strong $\mathrm{CO}_{2}$ absorption line. These samples were tested under two pumping regimes, via the co-doped $\mathrm{Yb}^{3+}$ ion coupled with $\mathrm{Er}^{3+}$ absorption around $980 \mathrm{~nm}$, and, in-band (or resonant) pumping of the $\mathrm{Er}^{3+}$ ion directly around $1480 \mathrm{~nm}$. The respective gain spectrum in the 1.5micron band was investigated, showing $>3 \mathrm{dBcm}^{-1}$ gain around the peak emission wavelength of $1531 \mathrm{~nm}$ from a sample having a concentration of both erbium and ytterbium ions of only $\sim 1.5 \times 10^{20} \mathrm{~cm}^{-3}$. Around the target wavelength of $1572 \mathrm{~nm}$ the gain depended upon the pumping wavelength and the glass' phonon spectrum, with $2.1 \mathrm{dBcm}^{-1}$ and $0.4 \mathrm{dBcm}^{-1}$ observed for the $974 \mathrm{~nm}$ and $1480 \mathrm{~nm}$ pump wavelengths, respectively.

Employing ceramic multi-component sputtering targets, composed of the respective oxides according to the recipe of the best glass composition trialled, doped and un-doped planar waveguides were subsequently fabricated via Radio Frequency (RF) magnetron and Ion-Beam Sputtering (IBS) methods. Oxidised silicon wafers (4" in diameter) were used as the substrate for the thin Tellurite films. Characterisation of the as deposited waveguide films has been made, providing information about the propagation losses, refractive index of layers, and surface roughness. Initial tests of the optical characteristics of the doped films are in progress. Furthermore we have produced multilayer waveguides, to make a pump guiding (effectively double-clad) structure that will cater for high-power pump guiding, with one example having a near single-mode $\sim 4$ micron thick core waveguide at the desired wavelength with a Numerical Aperture (NA) of 0.28 . The pump waveguide was $\sim 13 \mu \mathrm{m}$ thick and due to the high index contrast between the tellurite and $\mathrm{SiO}_{2}$ oxide layer has an NA>1. These structures are compatible with the aims of producing high power waveguide amplifiers for the wavelength regime of interest.

“jim@orc.soton.ac.uk; phone +44 238059 4521, fax +44 238059 3142, www.orc.soton.ac.uk 


\section{METHODOLOGY}

\subsection{Materials selection}

Tellurites glasses are known for their broad emission characteristics and high emission cross sections when doped with erbium [3], and therefore are expected to have a high figure of merit applicable to the wavelength range of interest for the measurement of $\mathrm{CO}_{2}$ gases. Typically Tellurite glasses are considered a low-phonon energy host for rare earths, with the vibrational mode energy spectrum confined to $\hbar \omega_{\max }<800 \mathrm{~cm}^{-1}$. Generally this means that if there is significant population in the ${ }^{4} \mathrm{I}_{11 / 2}$ energy level of $\mathrm{Er}^{3+}$, its modest lifetime could enable back-transfer of the excitation energy to the $\mathrm{Yb}^{3+}$ co-dopant. In 2012 [4] we explored multi-component Tellurite compositions, which would favour energy transfer from $\mathrm{Yb}^{3+}$ to $\mathrm{Er}^{3+}$ and rapidly de-excite the ${ }^{4} \mathrm{I}_{11 / 2}$ energy level of the latter, based on a zinc oxide glass former, magnesium oxide glass modifier, and a borate additive to capitalise on the more energetic stretching vibrations of the $\mathrm{B}-\mathrm{O}^{-}$bonds, up

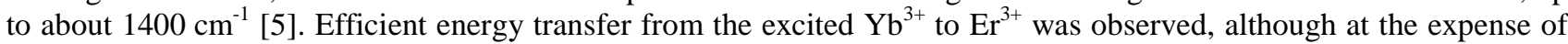
the ${ }^{4} \mathrm{I}_{13 / 2}$ manifold lifetime as well, $\tau_{\mathrm{f}} \sim 3 \mathrm{~ms}$. When proceeding to thin film fabrication we reverted to a composition without the borate modifier, i.e. $80 \mathrm{TeO}_{2}-10 \mathrm{ZnO}-10 \mathrm{MgO}(\mathrm{TZM})$, and it's $\mathrm{Er} / \mathrm{Yb}$ co-doped equivalent. In this way we capitalised on the low phonon energy host, maximizing the radiative lifetime of the ${ }^{4} \mathrm{I}_{13 / 2}$ energy level, measured to be $\tau_{\mathrm{f}} \sim 8.4 \mathrm{~ms}$, whilst still keeping the high gain characteristics. Moreover, we had intended to focus on in-band pumping, where the ${ }^{4} \mathrm{I}_{11 / 2}$ energy level would not be significantly populated, apart from excited state energy transfer processes.

\subsection{Bulk sample gain measurements}

Two sets of pump-probe gain measurements were made with this multi-component Er,Yb:TZM bulk sample. Firstly we employed a $500 \mathrm{~mW}$ fibre-coupled diode laser operating at $1480 \mathrm{~nm}$ (Furukawa electric FOL1435R50-617-1480), and secondly a similar $750 \mathrm{~mW}$ pump operating at $974 \mathrm{~nm}$ (Oclaro LC96UF74-20R). Both of these lasers were driven in a pulsed-mode by an arbitrary waveform function generator (Tektronix AFG 3102), producing 10 ms pulses at a 5\% duty cycle, thus avoiding any detrimental thermo-optical effects. The collimated fibre-coupled diode laser output was reflected by a high-pass dichroic filter and re-imaged into the sample with a beam diameter of $100(130) \mu \mathrm{m}$ for the respective $1480 \mathrm{~nm}(980 \mathrm{~nm})$ pumps, as illustrated in Figure 1. A CW tunable diode source (Tunics Plus CL/WB) was used as the probe, the single mode fibre output was also reimaged into the sample, in the same direction and collinear with the pump, and with a matched beam waist to that of the pump. The wavelength of the probe could be set between 1520-1600 nm with power levels up to $3 \mathrm{~mW}$. For the in-band pumped measurement the probe and residual pump beams were separated using a 600 lines/mm diffraction grating. The probe beam was then reimaged onto a large area Germanium detector (Thorlabs Model: SM1PD5B), covered by a $3 \mathrm{~mm}$ uncoated silicon window, while the transmitted pump was focused down onto an InGaAs detector (Thorlabs DET10D-M). Due to the larger wavelength difference between the $974 \mathrm{~nm}$ pump and $1.5 \mathrm{x} \mu \mathrm{m}$ probe, for that measurement a dichroic mirror was use to separate the pump/signal beams, and a large area silicon photo-detector was used to monitor the residual pump. Each photodiode output was recorded on a digital oscilloscope (Agilent MSO6104), during the pumping cycle and the change in signal power monitored for a number of discrete probe wavelengths across the available tuning range. The net gain for each sample was then calculated at various pump intensities for both pump wavelengths with respect to the un-pumped probe power prior to the start of the excitation pulse.

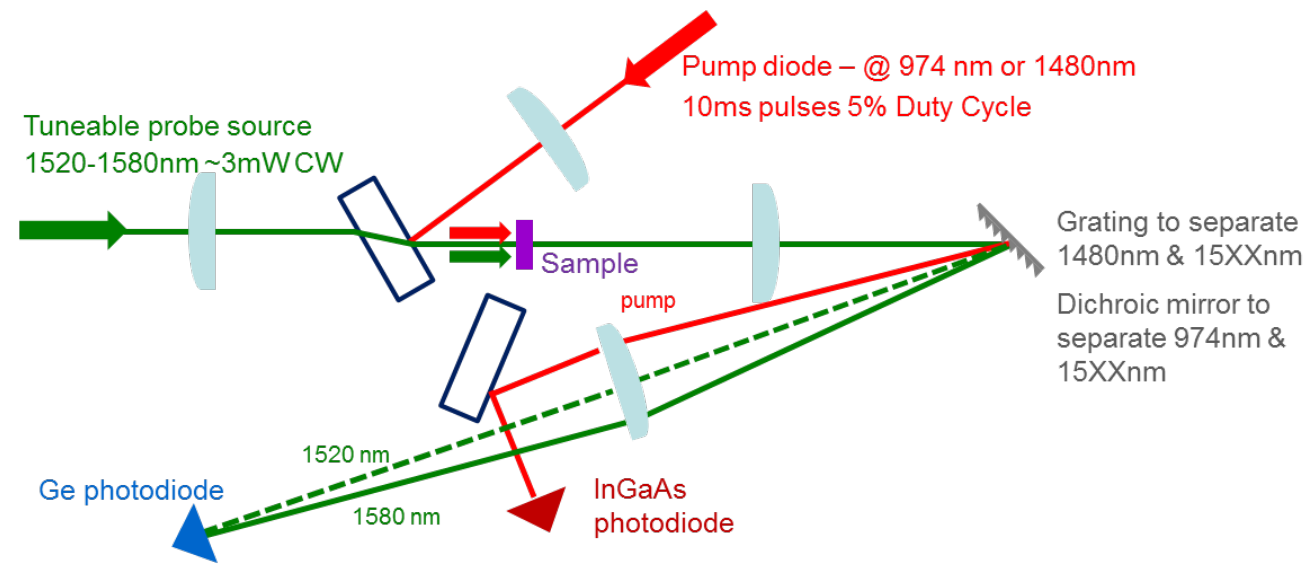

Figure 1. Gain measurement setup, as configured for the in-band pump-probe setup. 


\subsection{Fabrication and characterization of the thin films}

Two techniques were explored for fabricating thin films of the multi-component TZM glass. The first RF magnetron sputtering, employing an OPT Plasmalab400 system, and the second, IBS using an OPT Ionlab 300 Plus system. Both of these systems require 6" diameter targets of around $5 \mathrm{~mm}$ thickness. Two targets, with a molar composition of $80 \mathrm{TeO}-$ $10 \mathrm{ZnO}-10 \mathrm{MgO}$, were purchased from a commercial vendor made via a hot-press technique, one of which was codoped with $1.5 \mathrm{wt} \% \mathrm{Er}_{2} \mathrm{O}_{3}$ and $1.5 \mathrm{wt} \% \mathrm{Yb}_{2} \mathrm{O}_{3}$.

The substrates for the films were $1 \mathrm{~mm}$ thick, 4 ” silicon wafers with a 2.5 micron thermal oxide layer. Room temperature deposition was performed in a partial pressure atmosphere, with varied parameters for the respective machines and ratios of argon and oxygen flowing gases. After deposition, the thin films were characterized using a prism coupling system (Metricon - Model 2010) or ellipsometer system (J.A. Woollam M-2000) for their thickness and refractive index. In addition the surface quality and topology were measured via white light interferometry (Zemetrics - Zescope).

\section{RESULTS AND DISCUSSION}

\subsection{Bulk sample gain measurements}

Measurement of the single-pass small signal gain of the Er,Yb:TZM glass was made at discrete wavelengths (5nm steps from 1530-1565 nm, then 1nm steps between 1567-1577 nm). For the $980 \mathrm{~nm}$ pump, at a pump irradiance of $8 \mathrm{kWcm}^{-2}$ and near-normal incidence on the uncoated sample, the highest observed gain was at $1535 \mathrm{~nm}$ with $3.4 \mathrm{dBcm}^{-1}$, while at $1572 \mathrm{~nm}$ it was $2.1 \mathrm{dBcm}^{-1}$. With in-band pumping at $1480 \mathrm{~nm}$ the gain was significantly lower, even though at the pump irradiance of $6.1 \mathrm{kWcm}^{-2}$ where the theoretical inversion density, to fit the shortest wavelength data point, i.e. $\beta=0.68$, was the same as for the lowest pump irradiance of the $974 \mathrm{~nm}$ pump, i.e. $3 \mathrm{kWcm}^{-2}$. The temporal evolution of the probe beam power associated with the $10 \mathrm{~ms}$ pump pulse was captured at each probe wavelength, sometimes increasing at the front-end of the pulse then decaying toward the rear as previously observed in our earlier investigations of a similar multi-component composition[4]; revealing excited state energy transfer processes, such as Excited State Absorption (ESA). Figure 2 illustrates the measured gain corresponding to the two pump wavelengths, along with a simple gain calculation dependent upon the inversion factor, $\beta$, between the ground and the ${ }^{4} \mathrm{I}_{13 / 2}$ energy manifold. The $974 \mathrm{~nm}$ pump experiences stronger absorption and has a lower saturation-irradiance, $\mathrm{I}_{\mathrm{sat}} \sim 1.5 \mathrm{kWcm}^{-2}$, than the in-band pumping wavelength, i.e. $\mathrm{I}_{\mathrm{sat}} \sim 3 \mathrm{kWcm}^{-2}$ at $1480 \mathrm{~nm}$, and as such creates a higher inversion and therefore higher gain. However, the lifetime of the ${ }^{4} \mathrm{I}_{11 / 2}$ level is sufficiently long to allow a strong ESA transition, assisted by the excitation of two vibrational modes, promoting an $\mathrm{Er}^{3+}$ ion to its ${ }^{4} \mathrm{~F}_{9 / 2}$ energy state, leading to reduced gain, or even additional loss, at the longer probe wavelengths, Figure 2(a). In contrast, there is a small window around $1572 \mathrm{~nm}$ where there is a fortuitous gap between the symmetric and antisymmetric vibrations of the Te-O-Te linkages in $\mathrm{TeO}_{4}$ trigonal bi-pyramids at $\sim 550 \mathrm{~cm}^{-1}[6]$, reducing the effectiveness of the phonon assisted ESA loss and providing $>2 \mathrm{dBcm}^{-1}$ gain.

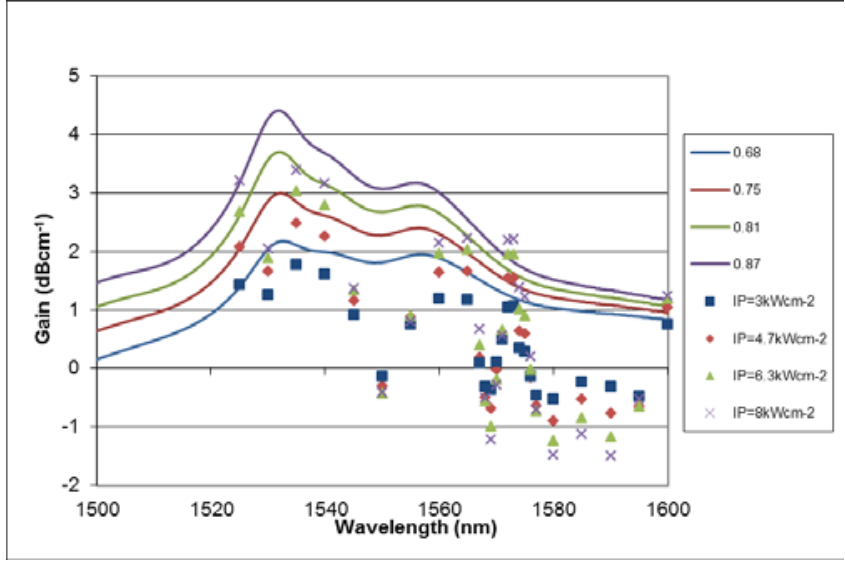

(a)

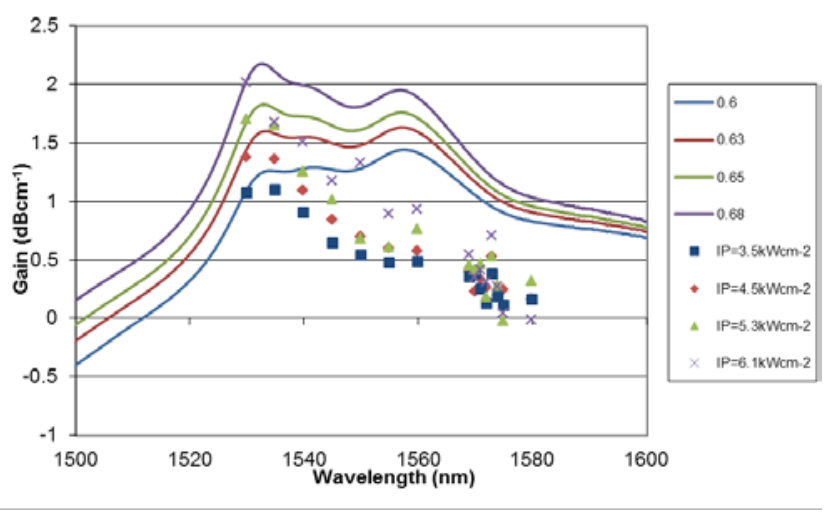

(b)

Figure 2. Theoretical and experimental gain in $\mathrm{dBcm}^{-1}$ for an Er,Yb:TZM bulk sample with (a) $974 \mathrm{~nm}$ and (b) $1480 \mathrm{~nm}$ pumping. Legend shows population inversion factor, $\beta$, used to estimate the expected gain level. 
Instead for the in-band pumped gain spectrum, Figure 2(b), there appears to be a different ESA process, from ${ }^{4} \mathrm{I}_{13 / 2}$ to ${ }^{4} I_{9 / 2}$, where one vibrational phonon is excited along with the absorption of a photon with a wavelength that covers pretty much the entire investigated range. It is noteworthy that there is a small spike around $1572 \mathrm{~nm}$, which again corresponds to the gap in the vibrational modes around $550 \mathrm{~cm}^{-1}$. Clearly as the regions of net loss, shown in Figure 2(a), are significantly stronger than in Figure 2(b), the coupling strength for the transition,

$$
E r^{3+},{ }^{4} I_{11 / 2}+h v_{\text {probe }}-2 h v_{\text {phonon }} \rightarrow E r^{3+},{ }^{4} F_{9 / 2}
$$

is much stronger than for,

$$
E r^{3+},{ }^{4} I_{13 / 2}+h v_{\text {probe }}-h v_{\text {phonon }} \rightarrow E r^{3+},{ }^{4} I_{9 / 2}
$$

This is consistent with the relative line-strengths for the respective ${ }^{4} \mathrm{~F}_{9 / 2}$ and ${ }^{4} \mathrm{I}_{9 / 2}$ energy manifolds. In addition it highlights the potential to tailor the glass composition to ensure that energy manifold positions and the phonon spectrum are optimal for this wavelength of interest for $\mathrm{CO}_{2}$ absorption spectroscopy.

\subsection{Thin films and characteristics}

The thin films from both of the fabrication techniques were characterized for their surface roughness. This is illustrated in Figure 3, showing that the deposited films are very smooth for both sputtering systems. Typical values for the root mean square surface area roughness, $\mathrm{S}_{\mathrm{q}}$, is on the order of $0.3-0.5 \mathrm{~nm}$ over an area of $\sim 6500 \mu \mathrm{m}^{2}$, as determined from white light interferometry. Additionally there are very few particulates and with a larger scale sampling, e.g. an area of $\sim 5.5 \mathrm{~mm}^{2}, \mathrm{~S}_{\mathrm{q}}$ values between $\sim 2-6 \mathrm{~nm}$ are typical. There is generally a slightly lower $\mathrm{S}_{\mathrm{q}}$ value for the IBS films compared to the RF sputtered films, however, the deposition rate is nearly an order of magnitude lower, i.e. up to $\sim 190 \mathrm{~nm} / \mathrm{hr}$ (IBS) compared to $\sim 1900 \mathrm{~nm} / \mathrm{hr}$ (RF) for the un-doped targets and about two thirds of this rate for the doped targets.

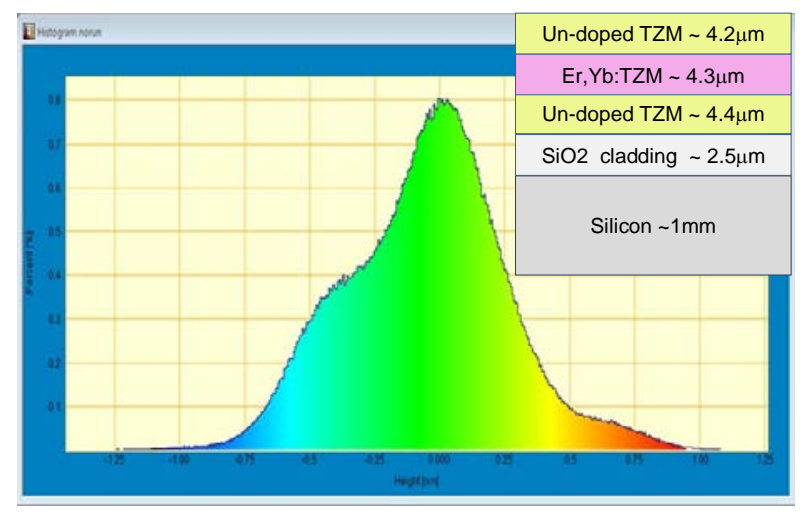

(a)

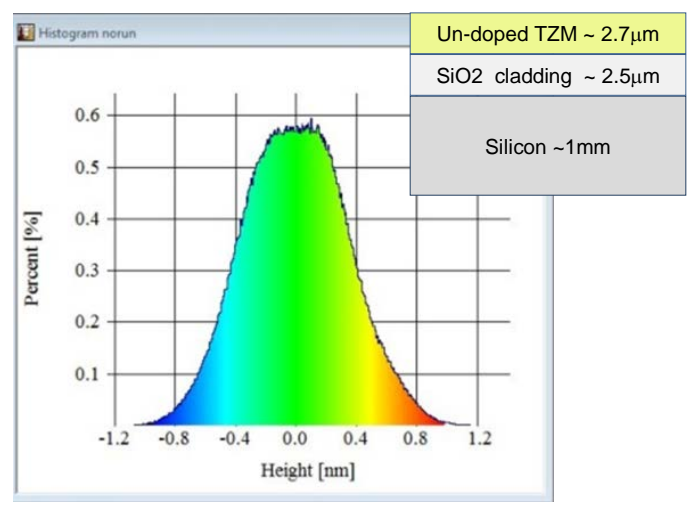

(b)

Figure 3: Example histograms for the surface roughness (at 100x magnification) for the (a) RF sputtered and (b) IBS films

Dark line prism coupling is a very effective tool to determine the waveguide properties, such the number of modes supported, the film refractive index and thickness, and the propagation losses. An example picture of one of the RF sputtered films is shown in Figure 4 (a), showing the coupled light reaching the edge of the wafer, along with the corresponding optical modes determined for the two polarization states for the $4.6 \mu \mathrm{m}$ thick TZM film, Figure 4(b). The refractive index of this film was found to be 1.96 at $633 \mathrm{~nm}$, significantly lower than that of the bulk glass sample measured to be 2.09. The propagation loss for this film was not measured; however, similar films had loss values between $1.2-2.1 \mathrm{dBcm}^{-1}$ as deposited, again at a wavelength of $633 \mathrm{~nm}$. Further post processing studies are about to be explored to see if this is an intrinsic loss of the material and if the waveguides can be improved.

Measurement of the refractive index of the multi-layer film illustrated in Figure 3(a) was also done with an ellipsometer, the result shown in Figure 5(a), alongside the refractive index measured for the bulk glass material in Figure 5(b). It is evident that the refractive index of the respective layers is lower than the bulk glass sample, and this is assumed to be due to the non-stoichiometric transfer of the target material to the sample. This will be tested via measuring the composition with energy dispersive $\mathrm{X}$-ray spectroscopy in the near future. 


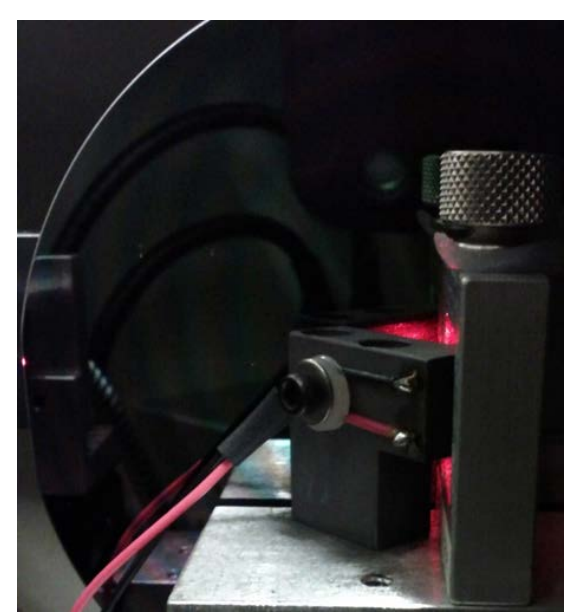

(a)

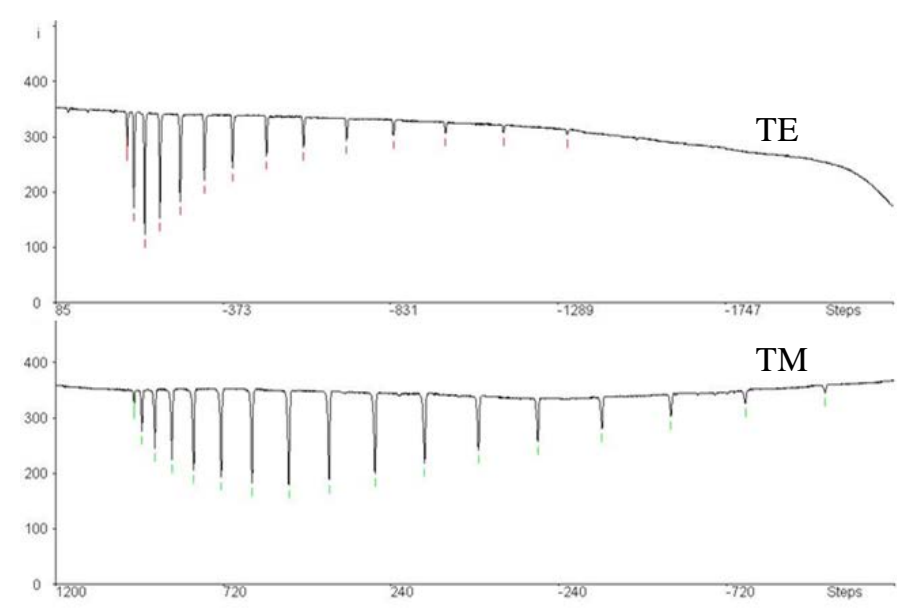

(b)

Figure 4: Dark-lines prism coupling setup with RF sputtered TZM film. (b) optical modes of the film providing the film thickness of $4.6 \mu \mathrm{m}$ and refractive index of 1.96 at $633 \mathrm{~nm}$.

To test that the erbium dopant is optically active we have measured the fluorescence spectrum of several samples, as illustrated in Figure 6. For different process gas ratios, i.e. argon to oxygen flow rates in the deposition chamber during sputtering, we see quite a different shape in the emission spectra. Again this indicates that the film composition depends heavily on the growth conditions, which have been tabulated in Table 1 for the three variations shown in Figure 6. The broadest spectrum comes from the multi-layer stack illustrated in Figure 3, and visually in the photograph inset of Figure 6, you can see the signature up-converted emission in the green, characteristic of strongly pumped $\mathrm{Er}^{3+}$.

Table 1: Process parameters for waveguides used for the fluorescence studies

\begin{tabular}{c|c|c|c|c|c|c|c|c|c}
\hline \hline SN & Taget & $\begin{array}{c}\text { Duratio } \\
\mathrm{n}(\mathrm{hrs})\end{array}$ & $\begin{array}{c}\text { Dep } \tau \\
(\mathrm{mTorr})\end{array}$ & $\begin{array}{c}\mathrm{Ar} \\
(\mathrm{sccm})\end{array}$ & $\begin{array}{c}\mathrm{O}_{2} \\
(\mathrm{sccm})\end{array}$ & $\mathrm{Ar}_{2} \mathrm{O}_{2}$ & $\begin{array}{c}\mathrm{n} \\
(633 \mathrm{~nm})\end{array}$ & $\mathrm{t}(\mu \mathrm{m})$ & $\begin{array}{c}\text { Dep rate } \\
(\mathrm{nm} / \mathrm{hr})\end{array}$ \\
\hline 33 & ErYbTMZ & 3 & 7.5 & 20 & 5 & 4 & 1.9555 & 3.105 & 1035.0 \\
34 & ErYbTMZ & 3 & 7.5 & 8 & 16 & 0.5 & 1.9397 & 1.8842 & 628.1 \\
38 & $\begin{array}{c}\text { TZM/Er,Yb:TZM } \\
\text { /TZM }\end{array}$ & 11 & 10 & & & & & $\sim 13$ & $\sim 1200$
\end{tabular}

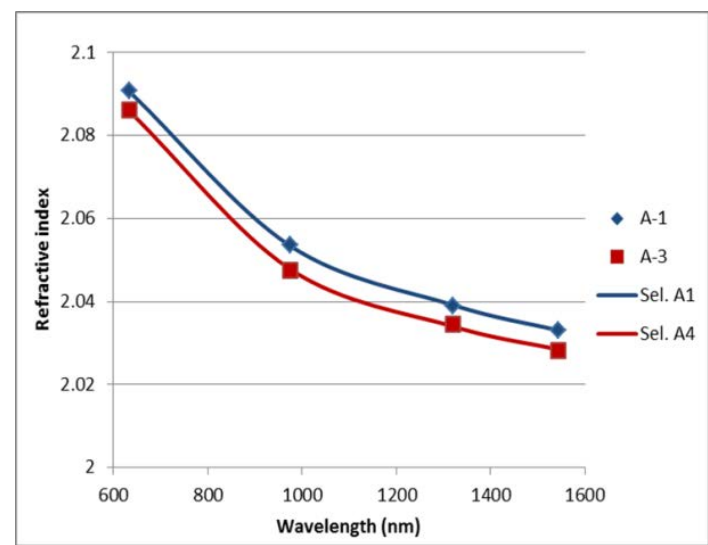

(a)

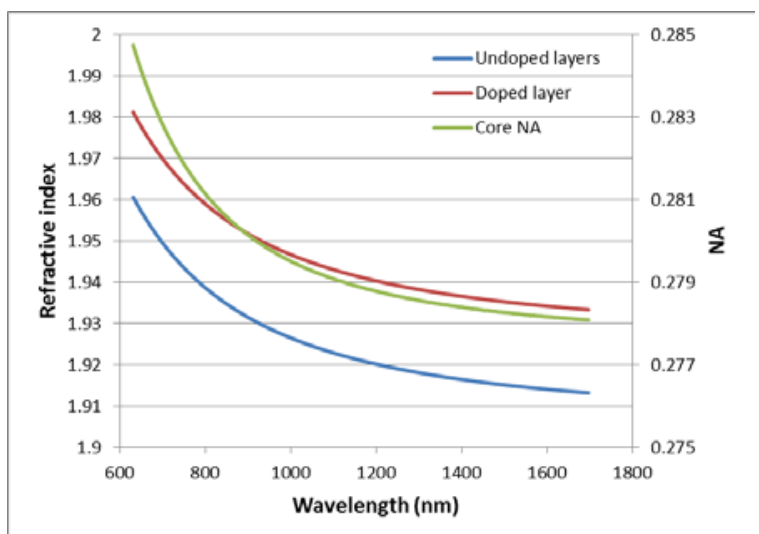

(b)

Figure 5: (a) refractive index of the TMZ (A-1) and Er,Yb:TZM (A-3) bulk glass samples and the Sellmeier equation fit, and

(b) the ellipsometer measured refractive index of the multi-layer stack of Figure 3(a). 


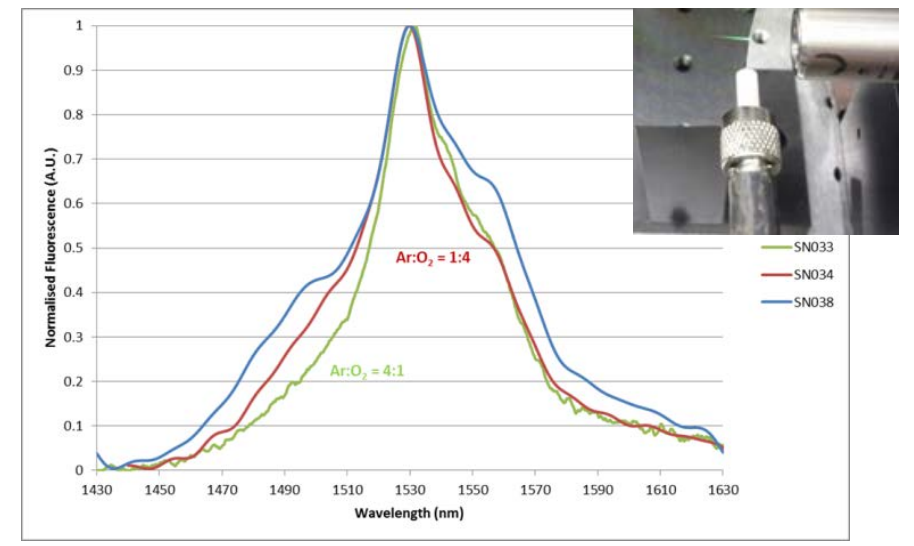

Figure 6: Normalized fluorescence spectrum for different samples and process gas ratios. SN038 is the sample illustrated in Figure 3(a).

\section{CONCLUSION}

We have reported the fabrication and characterization of erbium/ytterbium co-doped and undoped tellurite waveguide films, targeting power amplification in the wavelength band around the $1572 \mathrm{~nm} \mathrm{CO}$ absorption line. In the starting bulk glass sample, small signal gain coefficients in excess of $3 \mathrm{dBcm}^{-1}$ have been measured around the $1531 \mathrm{~nm}$ emission peak and $>2 \mathrm{dBcm}^{-1}$ at $1572 \mathrm{~nm}$, when pumped with a diode laser operating at $974 \mathrm{~nm}$. The excellent surface quality of these films, the ability to grow "double-clad" structures, and the underlying advantageous thermal properties of the silicon platform support the potential for these waveguides to be operated at high average powers.

\section{ACKNOWLEDGEMENTS}

The authors would like to thank NASA ESTO and the Engineering and Physical Sciences Research Council (EPSRC), grant number EP/J008052/1, for their financial support of this effort.

\section{REFERENCES}

[1] Munoz-Martin, D., Fernandez, H., Fernandez-Navarro, J. M. et al., "Nonlinear optical susceptibility of multicomponent tellurite thin film glasses,” Journal of Applied Physics, 104(11), 113510-1 (2008).

[2] Madden, S. J., and Vu, K. T., "High-Performance Integrated Optics with Tellurite Glasses: Status and Prospects,” International Journal of Applied Glass Science, 3(4), 289-298 (2012).

[3] Mori, A., Ohishi, Y., and Sudo, S., "Erbium-doped tellurite glass fibre laser and amplifier,” Electronics Letters, 33(10), 863-864 (1997).

[4] Mackenzie, J. I., Murugan, G. S., Suzuki, T. et al., "Investigation of erbium-doped tellurite glasses for a planar waveguide power amplifier at $1.57 \mu \mathrm{m} "$, in "Proc. SPIE 823514, (2012).

[5] Maniu, D., Iliescu, T., Ardelean, I. et al., "Raman study on $\mathrm{B}_{2} \mathrm{O}_{3}-\mathrm{CaO}$ glasses,” Journal of Molecular Structure, 651, 485-488 (2003).

[6] Murugan, G. S., and Ohishi, Y., "Structural and physical properties of a novel $\mathrm{TeO}_{2}-\mathrm{BaO}-\mathrm{SrO}-\mathrm{Ta}_{2} \mathrm{O}_{5}$ glass system for photonic device applications,” Journal of Non-Crystalline Solids, 351(5), 364-371 (2005). 